\title{
Equinococosis quística músculo-esquelética primaria de evolución crónica
}

\author{
Eduardo Botello', Cristián Ruz', Carolina Avilés², Sebastián Valderrama³, Marisa Torres ${ }^{4}$
}

'Departamento de Ortopedia y Traumatología, Pontificia Universidad Católica de Chile. ${ }^{2}$ Departamento de Ortopedia y Traumatología, Facultad de Medicina, Clínica Alemana

Universidad del Desarrollo ${ }^{3}$ Departamento de Medicina Interna, Pontificia Universidad Católica de Chile. ${ }^{4}$ Departamento de Salud Pública Facultad de Medicina, Pontificia Universidad Católica de Chile.

Conflicto de interés: ninguno. Fuente de financiamiento: ninguna.

Recibido: 21 de diciembre de 2017 Aceptado: 13 de noviembre de 2018

\section{Introducción}

L a equinococosis quística o hidatidosis es una zoonosis parasitaria producida por la larva del cestode Echinococcus granulosus. Es una infección re-emergente de amplia distribución mundial ${ }^{1}$, que integra el grupo de las enfermedades que la Organización Mundial de la Salud ha denominado enfermedades tropicales desatendidas (ETD) ${ }^{2-5}$. Echinococcus granulosus sensu stricto es la principal causa de equinococosis quística en la mayoría de los casos humanos y animales en el mundo y la especie más extendida dentro del complejo E. granulosus sensu lato. Echinococcus granulosus s.s. es endémico en América del Sur junto con otras especies del género Echinococcus, especialmente en algunas áreas en Argentina, Brasil, Chile y Perú.

En Chile, la equinococosis quística está presente en todo el territorio nacional (excepto en la Antártida y Rapa Nui) y es causada por E. granulosus cepa oveja. Un estudio nacional reciente de 69 muestras parasitarias identificó 21 haplotipos del parásito no descritos, evidenciando la variabilidad parasitaria existente en el territorio ${ }^{6}$. En Chile, la tasa de infección por E. granulosus de acuerdo al Sistema de Vigilancia de Enfermedades de Notificación Obligatoria (SVE-ENO) es de 1,48 casos por 100.000 habitantes, la que presenta gran variación según la región ${ }^{7}$. Se reconoce una sub-notificación de la enfermedad por parte de los equipos tratantes, ya que las tasas de egresos hospitalarios por esta causa son mayores a los casos notificados ${ }^{8,9}$ (7.364 días de hospitalización en el año 2015, con 11,8 días en promedio) (Tabla 1). La frecuencia de localización varía de acuerdo al grupo etario, siendo el hígado y pulmón los órganos afectados más frecuentes (Tabla 2).

Aunque las tasas nacionales han descendido en las últimas décadas, actualmente existen regiones con mayor endemicidad, como Coquimbo, Araucanía y Aysén, donde se están implementando programas de control local ${ }^{10,11}$. La equinococosis quística humana genera altos costos sociales y económicos, por atención médica (hospitalizaciones, terapia, cirugía), ausentismo laboral y escolar.

La localización primaria de hidátides en el aparato músculo-esquelético es infrecuente y de difícil diagnósti$\mathrm{co}^{12-14}$. Su tratamiento está poco estandarizado y existe escasa evidencia sobre la terapia quirúrgica y farmacológica. Presentamos un caso de equinococosis quística musculoesquelética de rara ocurrencia y de evolución crónica.

\section{Caso clínico}

Varón de 66 años, profesional dedicado a labores administrativas, residente de una zona urbana de la Región del Maule, Chile, zona endémica que en la actualidad no cuenta con programas de control para esta zoonosis ${ }^{15}$. Tenía antecedentes de obesidad leve, hipertensión arterial en etapa I, diabetes mellitus tipo II e hiperplasia prostática benigna, patologías crónicas en control con tratamiento farmacológico con enalapril, atorvastatina y metformina. Refería haber vivido en una zona rural en su infancia y 


\begin{tabular}{|c|c|c|}
\hline \multirow[t]{2}{*}{ Equinococcosis (B67) } & \multicolumn{2}{|c|}{ Días de estada } \\
\hline & $\begin{array}{c}\text { Número } \\
7.364\end{array}$ & $\begin{array}{c}\text { Promedio } \\
11,8\end{array}$ \\
\hline Infección del hígado debida a Echinococcus granulosus (B670) & 736 & 12,7 \\
\hline Infección del pulmón debida a Echinococcus granulosus (B671) & 384 & 12,4 \\
\hline Infección de hueso debida a Echinococcus granulosus (B672) & - & - \\
\hline Infección de otro órgano y de sitios múltiples debida a Echinococcus granulosus (B673) & 139 & 17,4 \\
\hline Infección debida a Echinococcus granulosus, sin otra especificación (equinococosis) (B674) & 20 & 20,0 \\
\hline Infección del hígado debida a Echinococcus multilocularis (B675) & - & - \\
\hline Infección de otro órgano y de sitios múltiples debida a Echinococcus multilocularis (B676) & - & - \\
\hline Equinococosis del hígado, no especificada (B678) & 2.835 & 10,7 \\
\hline Equinococosis, otra y la no especificada (B679) & 3.250 & 12,4 \\
\hline
\end{tabular}

\begin{tabular}{|c|c|c|c|c|c|c|c|c|c|c|}
\hline Órgano afectado & $<1$ a & $1-4$ & $5-9$ & $10-14$ & $15-19$ & $20-44$ & $45-64$ & $65-79$ & $80 y+$ & Total \\
\hline Echinococosis total (B 670) & - & 10 & 42 & 33 & 45 & 229 & 216 & 100 & 25 & 700 \\
\hline Hígado (B 670) & - & - & 7 & 3 & 4 & 24 & 18 & 81 & 1 & 65 \\
\hline Pulmón (B 671) & - & 2 & 4 & 1 & 5 & 10 & 13 & 4 & 2 & 41 \\
\hline Hueso (B672) & - & - & - & - & - & - & - & 1 & - & 1 \\
\hline Otro órgano o múltiple (B676) & - & - & - & 1 & - & 4 & 1 & 3 & - & 9 \\
\hline Sin otra explicación & - & - & - & - & - & - & - & 1 & - & 1 \\
\hline Equinococosis del hígado, no especificada & - & 2 & 11 & 17 & 18 & 99 & 98 & 34 & 14 & 293 \\
\hline No especificada (B 679) & - & 6 & 20 & 11 & 18 & 92 & 86 & 49 & 8 & 290 \\
\hline
\end{tabular}

viajado a zonas urbanas de Europa hacía tres años. No tenía perros, no presentaba geofagia u onicofagia, no realizaba actividades agrícolas o de jardinería, ni frecuentaba zonas rurales en forma habitual.

El paciente presentaba un historia de cuatro años de dolor insidioso lumbosacro izquierdo, con múltiples consultas, tratamientos médicos (analgesia oral, tópica e infiltraciones locales), sin respuesta clínica. Hacía ocho meses, se agregó dolor y aumento de volumen progresivo de la cara lateral del muslo izquierdo. Se había realizado una ecografía y radiografía de la zona lumbosacra que sugerían un hematoma y probables lesiones líticas secundarias; respectivamente. Esto motivó su consulta en la red de salud de Santiago. Al examen físico destacaba una importante limitación funcional de la extremidad inferior izquierda, con aumento de temperatura, eritema local y una fístula cutánea en el muslo izquierdo, que daba salida a líquido cristalino (Figura 1). Se realizó una tomografía computada (TC) de tórax, abdomen y pelvis que evidenció la presencia de múltiples lesiones quísticas

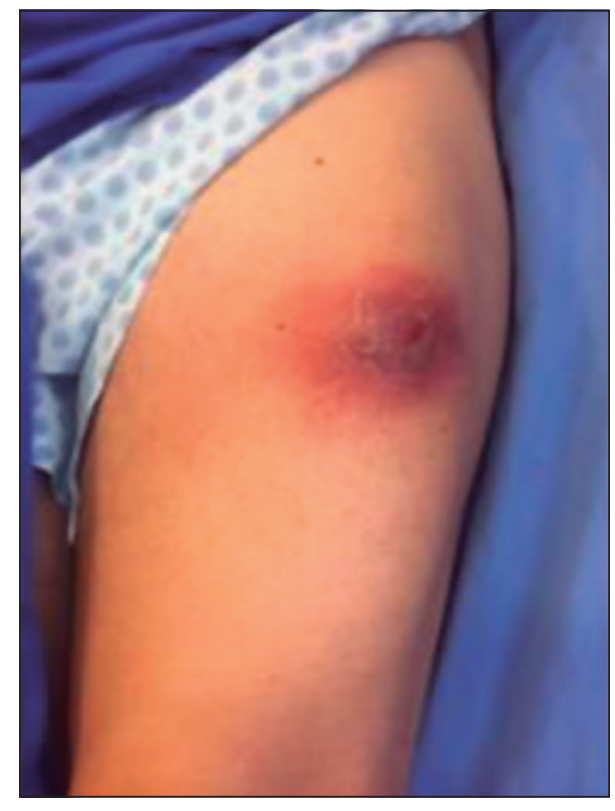

Figura 1. Cara anterolateral de muslo izquierdo con equinococosis quística. 


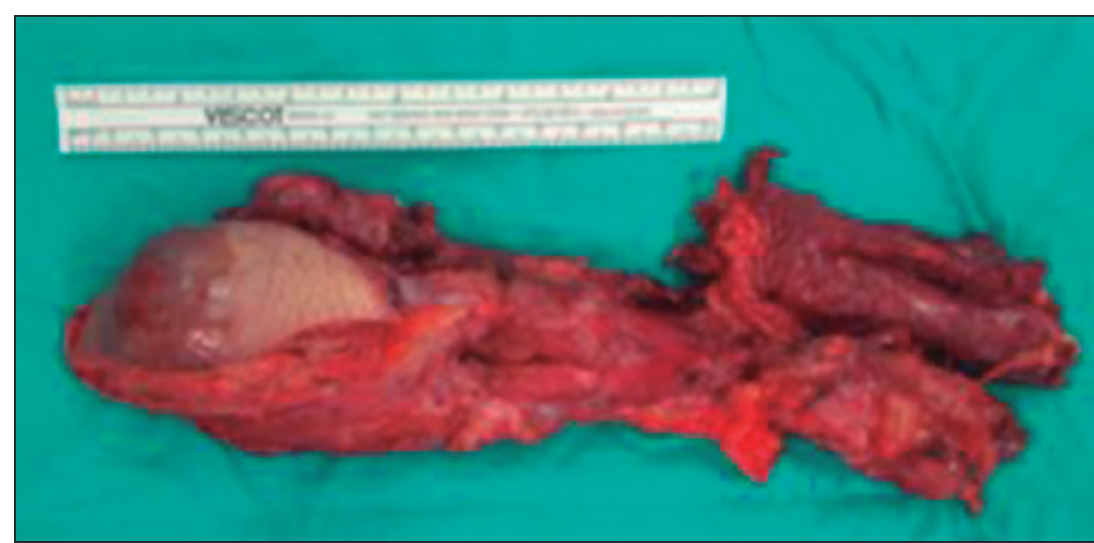

Figura 2. Pieza quirúrgica. Resección amplia de múltiples quistes de muslo.

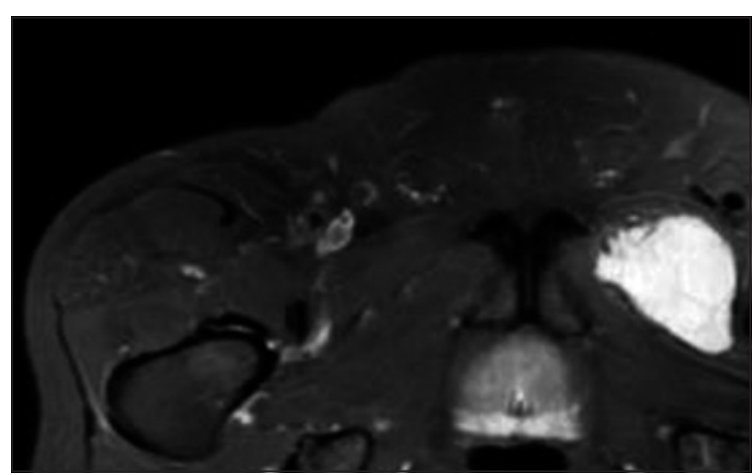

Figura 3. RM secuencia Stir, axial. Múltiples quistes de tejidos blandos de zona inguinal y región peritrocantérica izquierda.

Figura 4. RM secuencia Stir, coronal. Múltiples lesiones de tejidos blandos del compartimento lateral del muslo izquierdo. intraóseas que comprometían el hueso iliaco izquierdo, principalmente el acetábulo y ala ilíaca, extendiéndose a los tejidos blandos adyacentes, con compromiso del músculo obturador externo y pectíneo, alcanzando una longitud aproximada de 5,7 cms. Se observaba además, compromiso intramuscular de la cara anterior del muslo (vasto intermedio y lateral) y la porción iliaca del músculo psoas. Los hallazgos descritos en región iliaca y muslo proximal eran compatibles con una enfermedad hidatídica. Se descartó la presencia de quistes en hígado y pulmón. La serología para E. granulosus fue positiva (ELISA IgG), realizada en el Laboratorio de Parasitología del Instituto de Salud Pública, Centro de Referencia Nacional.

Se realizó una resección quirúrgica de tejidos blandos del muslo con un margen amplio (Figura 2). El estudio histopatológico informó un segmento de músculo estriado con áreas blanco-amarillentas granulares, zonas de necrosis y membranas café-amarillentas, transparentes de hasta $0,5 \mathrm{~cm}$ de longitud, que comprimían la musculatura y vainas tendinosas. El diagnóstico microscópico fue un quiste hidatídico no vital, roto e inflamado, con reacción granulomatosa del tejido fibroconectivo y muscular esquelético adyacente. No se realizó estudio de la cepa parasitaria.

Se informó al paciente sobre la enfermedad, su eventual curso clínico y pronóstico. Tanto el paciente como su familia desconocían la enfermedad, sus manifestaciones y sus factores de riesgo. Ante el compromiso pélvico masivo, la escasa posibilidad de controlar localmente la enfermedad pese a realizar una resección amplia de las lesiones, con alto riesgo de secuelas invalidantes, el equipo médico en conjunto con el paciente y su familia decidieron tratamiento conservador con fármacos antiparasitarios (albendazol y praziquantel). Se inició terapia antiparasitaria farmacológica con ciclos mensuales de albendazol oral (10 mg/kg/día, fraccionado en dos dosis), con descanso de una semana cada vez. Posteriormente se incorporó praziquantel $(10 \mathrm{mg} / \mathrm{kg} /$ día, una vez a la semana) por cuatro veces, con lo que se obtuvo remisión del cuadro clínico. Diez semanas después de la cirugía reapareció la fístula, la que fue resuelta de forma quirúrgica. Se realizó una resonancia magnética (RM) de pelvis y muslo de control que mostró lesiones de contenido quístico en tejidos blandos de la región inguinal y muslo (Figura 3, 4 y 5), compromiso de médula ósea en hueso iliaco, isquion y rama iliopubiana izquierda, compatible con hidatidosis e imágenes quísticas de ubicación yuxtacortical, compatibles con vesículas hidatídicas, sin variación al estudio previo. Sí se observó una significativa disminución del compromiso hidatídico en el muslo.

En el hemograma el paciente presentaba leucocitosis con eosinofilia absoluta (288 eosinofilos $/ \mathrm{mm}^{3}$. El paciente se controló clínicamente en forma mensual, o bimensual, con exámenes generales (hemograma, perfil bioquímico), 
y cada 12 meses con serología IgG E. granulosus en Instituto de Salud Pública.

A los ocho meses de tratamiento, el paciente ante ausencia de síntomas, decidió suspender la terapia antiparasitaria, con lo cual reaparecieron los síntomas y signos locales. El equipo tratante reinició la terapia farmacológica. Hasta la publicación de este reporte, el paciente permanece en terapia con albendazol y praziquantel. Se ha mantenido autovalente, realizando sus actividades laborales, con parámetros hematológicos y hepáticos normales.

\section{Discusión}

En este caso, la equinococosis quística ha tenido un comportamiento clínico crónico insidioso y prolongado. El diagnóstico específico se demoró años por su presentación atípica y poco frecuente. A petición del paciente y en beneficio de su salud integral, durante el proceso ha mantenido sus actividades laborales, lo que ha permitido mantener su calidad de vida.

La equinococosis quística músculo-esquelética es poco frecuente, de difícil diagnóstico y tratamiento. Esta presentación generalmente es local, no presenta compromiso visceral y puede manifestarse en cualquier hueso ${ }^{16}$; sin embargo, se observa con mayor frecuencia en columna vertebral, fémur y pelvis ${ }^{17}$. En la literatura médica existen escasos reportes de equinococosis de localización pelviana única, la mayoría series clínicas pequeñas o casos aislados.

Para el diagnóstico clínico de la equinococosis quística músculo-esquelética las técnicas por imagen constituyen un gran aporte, siendo la RM el examen de elección, pues aporta elementos relevantes para planificar la intervención quirúrgica ${ }^{18}$. El estudio serológico a través de técnicas de inmunodiagnóstico tiene baja sensibilidad y especificidad en casos de quistes hidatídicos extrahepáticos aislados ${ }^{19,20}$. Aun así, dado su bajo costo, esta técnica es utilizada en las primeras etapas del estudio clínico. El resultado serológico de inmunoglobulina $\mathrm{G}$ para $E$. granulosus apoya el diagnóstico, no obstante, si el resultado es negativo no descarta la infección.

En general, en equinococosis quística, la biopsia con técnica abierta de hidátides intactas está contraindicada por riesgo de diseminación y shock anafiláctico ${ }^{21}$. En este caso, dado su presentación lentamente progresiva y su difícil diagnóstico, la intervención quirúrgica fue tardía, lo que impidió erradicar el parásito.

De acuerdo a los expertos, el tratamiento de la equinococosis quística músculo-esquelética por consenso considera el uso de cirugía con resección con márgenes amplios $^{22-25}$ combinados con terapia farmacológica antihelmíntica; sin embargo, no hay revisiones sistemáticas

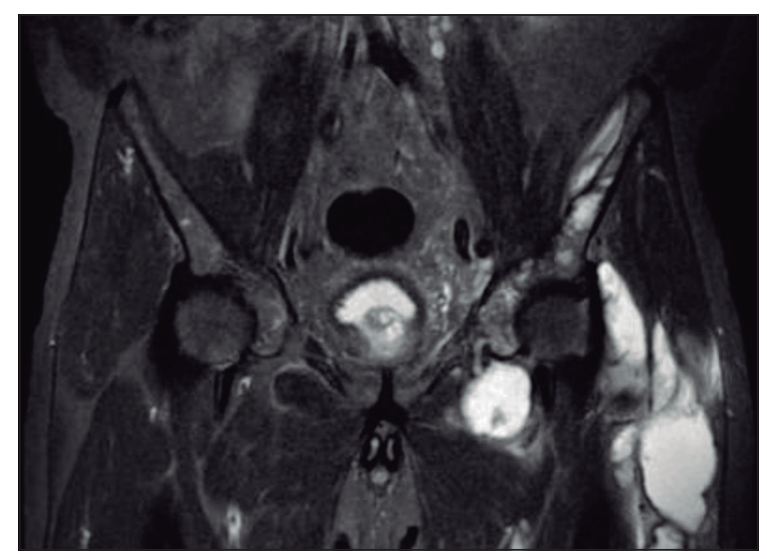

Figura 5. RM pelvis secuencia Stir, coronal. Extenso compromiso inflamatorio de tejidos blandos en cara lateral de muslo, ingle, acetábulo y ala ilíaca izquierda. que lo apoyen. El fármaco usado para la terapia en esta presentación clínica de primera línea es albendazol (10 $\mathrm{mg} / \mathrm{kg} / \mathrm{día})^{26}$. Algunas revisiones apoyan la terapia biasociada post-quirúrgica de albendazol y praziquantel, sin evidencia de lograr una mejor respuesta ${ }^{27}$. En este caso, la terapia local del muslo abordó las complicaciones de los quistes rotos, y la terapia farmacológica buscó controlar la enfermedad remanente.

En el contexto de las escasas opciones terapéuticas para la hidatidosis, es relevante mencionar que estudios recientes señalan que metformina, hipoglicemiante que recibía nuestro paciente, con potencial agente anticancerígeno, podría ejercer su efecto anti-proliferativo en hidatidosis vía inducción de estrés energético. En un estudio de eficacia in vitro e in vivo de metformina contra el estado larval de E. granulosus mostró efectos de muerte en parásitos en cultivos (protoscólices y metacestodos in vitro) que fueron significativos y dependientes de la dosis y el tiempo. La administración oral de metformina (50 $\mathrm{mg} / \mathrm{kg} / \mathrm{día}$ ) en ratones infectados con E. granulosus fue altamente efectiva para reducir el tamaño y la cantidad de quistes de parásitos; sin embargo, la acumulación de metformina intraquística fue mayor en los animales tratados con albendazol y metformina en comparación con los administrados con metformina sola. Estos autores señalan que, basados en sus datos, la metformina emerge como un fármaco anti-equinocócico prometedor ya que ha demostrado que inhibe eficientemente el desarrollo y el crecimiento de la etapa larvaria de E. granulosus y que su combinación con albendazol puede mejorar la terapia $\operatorname{antiparasitaria}^{28}$.

El tiempo de duración de la terapia de la hidatidosis musculo-esquelética es variable y depende de la evolución clínica y serológica ${ }^{29}$, con rangos que van desde los seis meses a los 10 años. La recurrencia de hidatidosis ósea es de $17 \%{ }^{30}$. La principal variable asociada al riesgo de recurrencia es el compromiso visceral.

El estudio y segumiento del paciente fue difícil por 
la distancia entre su domicilio y la red de atención del equipo tratante $(256 \mathrm{~km})$, por lo que finalmente se derivó al Hospital Regional de Talca. Otra limitación fue el no contar con estudio de la cepa parasitaria. Durante todo el curso de su enfermedad el paciente persistió con dolor crónico, el que ha requerido analgesia multimodal. En este caso, la hidatidosis se ha comportado como un proceso neoplásico, que no ha sido del todo controlado. Para prevenir casos como el presentado, en especial en Chile, país endémico, se debe enfatizar en la necesidad de contar con un Programa Nacional continuo y sustentable de control y prevención de la equinococosis quística.

\section{Conclusiones}

El diagnóstico y tratamiento de la equinococosis quística músculo-esquelética sigue siendo un desafío. $\mathrm{Su}$ sospecha debe ser alta en zonas endémicas como nuestro país. La serología ELISA IgG para equinococosis es muy útil para la hidatidosis hepática y pulmonar, pero tien baja sensibilidad y especificidad en localizaciones atípicas como el caso que se presenta. En estos casos la serología positiva apoya el diagnóstico clínico. La RM es la mejor técnica de imagen de estudio local y la TC ayuda a precisar la etapa del compromiso. El tratamiento se basa en la resección amplia de las lesiones y el apoyo farmacológico se constituye en un arma eficiente y necesaria para el control de la enfermedad, por lo que es importante evaluar nuevas terapias ${ }^{23,24}$.

\section{Resumen}

La equinococosis quística es una zoonosis parasitaria crónica de alta prevalencia en Chile. Se presenta el caso clínico de un varón de 66 años, proveniente de la Región del Maule, con una equinococosis quística músculoesquelética. Consultó por dolor, aumento de volumen y una fístula en muslo izquierdo, con salida de líquido cristalino. En el estudio imagenológico se identificaron múltiples lesiones quísticas en el ala sacra, hueso ilíaco y tejidos blandos de zona inguinal y muslo izquierdo. La serología Elisa IgG para Equinococcus granulosus fue positiva. Se realizó la resección quirúrgica de las lesiones musculares y se inició terapia antiparasitaria combinada con albendazol y praziquantel, con buena respuesta clínica; sin embargo, al suspender la terapia, por iniciativa del paciente, se reiniciaron los síntomas.

\section{Referencias bibliográficas}

1.- Álvarez Rojas C A, Fredes F, Torres M, AcostaJamett G, Alvarez J F, Pavletic C, et al. Erratum to: First meeting "Cystic echinococcosis in Chile, update in alternatives for control and diagnostics in animals and humans". Parasites \& Vectors 2016; 9: 502

2.- Kappagodaa S, Ioannidisb J P A. Prevention and control of neglected tropical diseases: overview of randomized trials, systematic reviews and meta-analyses. Bull World Health Organ 2014; 92: 356-66C.

3.- Parra-Valencia E, Urra-Canales A. Echinococcosis and cysticercosis: neglected diseases in Chile. Medwave 2016; 16: e6566. doi: 10.5867/medwave.2016.09.6566.

4.- Martínez P. Human hydatidosis in children: manifestation of failure in the control and prevention measures. Chile, 2001-2011 Rev Chilena Infectol 2015; 32: 158-66. doi: 10.4067/S0716-10182015000300004.

5.- Pavletic C, Larrieu E, Guamera E A, Casas $\mathrm{N}$, Irabedra P, Ferreira C, et al. Cystic echinococcosis in South America: a call for action. Rev Panam Salud Publica 2017; 41: e42.

6.- Alvarez Rojas C A, Ebi D, Paredes R, Acosta-Jamett G, Urriola N, Roa J C, et al. High intraspecific variability of Echinococcus granulosus sensu stricto in Chile. Parasitol
Int 2017; 66: 112-5. doi: 10.1016/j. parint.2016.12.001.

7.- Fuenzalida F. Hidatidosis (CIE 10: B67). Situación epidemiológica, semanas 1 a 52 , año 2012 (01/01/2012 a 29/12/2012). Dpto. de Epidemiología, DIPLAS-MINSAL. Norma Técnica $\mathrm{N}^{\circ} 55$ de Vigilancia de enfermedades.

8.- Serra I, Araya C, Araneda J. Evolución epidemiológica de la hidatidosis humana en Chile, 1965-1988. Impacto de programas ganaderos en las regiones XI y XII. Rev Med Chile 1983; 121: 343-9.

9.- Godoy K, Gálvez G, Tassara R. Frecuencia de hidatidosis en los principales centros quirúrgicos de Santiago de Chile, comparación con notificación al Ministerio de Salud (19881997). Bol Hosp San Juan de Dios 2000; 47: 309-13.

10.- Moro P, Schantz P M. Cystic echinococcosis in the Americas. Parasitol Int 2006; 55 (Suppl): S181-6.

11.- Schenone H, Contreras M C, Salinas P, Sandoval L, Saavedra T, Rojas A. Hidatidosis humana en Chile. Seroprevalencia y estimación del número de personas infectadas. Bol Chil Parasitol 1999; 54: 70-3.

12.- Merkle E M, Schulte M, Vogel J, Tomczak R, Rieber A, Kern P, et al. Musculoskeletal involvement in cystic echinococcosis: Report of eight cases and review of the literature. Am J Roentgenol 1997; 168: 1531-4.
13.- Manterola C, Otzen T. Hepatic echinococcosis with thoracic involvement. Clinical characteristics of a prospective series of cases. Ann Hepatol. 2017; 16: 599-606. doi: 10.5604/01.3001.0010.0305.

14.- Bracanovic D, Djuric M, Sopta J, Djonic D, Lujic N. Skeletal manifestations of hydatid disease in Serbia: demographic distribution, site involvement, radiological findings, and complications. Korean J Parasitol 2013; 51: 453-9. doi: 10.3347/kjp.2013.51.4.453

15.- Apt W, Pérez C, Galdámez E, Campano S, Vega $\mathrm{F}$, Vargas D, et al. Equinococosis/hidatidosis en la VII Región de Chile: diagnóstico e intervención educativa. Rev Panam Salud Publica/Pan Am J Public Health 2000; 7: 8-16.

16.- Zia S, Enam A, Salahuddin I, Khan A. Role of irrigation with hypertonic saline for a recurrent skull base hydatid cyst: case report and review of the literature. Ear Nose Throat J 2010; 89: 22-6.

17.- Ebrahimi A, Assadi M, Saghari M, Eftekhari M, Gholami A, Ghasemikhah R, et al. Whole body bone scintigraphy in osseous hydatosis: a case report. J Med Case Rep 2007; 1: 93.

18.- Singh S, Korah I P, Gibikote S V, Shyam N K, Nair A, Korula A. Sacral hydatidosis: value of MRI in the diagnosis. Skeletal Radiol 1998; 27: 518-21.

19.- Manzano-Román R, Sánchez-Ovejero C, Hernández-González A, Casulli A, Siles-Lucas 
M. Serological diagnosis and follow-up of human cystic echinococcosis: a new hope for the future? Biomed Res Int 2015: 428205. doi:10.1155/2015/428205.

20.- Marinova I, Nikolov G, Michova A, Kurdova R, Petrunov B. Quantitative assessment of serum-specific $\mathrm{IgE}$ in the diagnosis of human cystic echinococcosis. Parasite Immunol 2011; 33: 371-6. doi: 10.1111/j.13653024.2011.01292.x.

21.- Heinze J, Junginger W, Müller G, Gaebel G Anaphylactic shock during excision of an intraosseous Echinococcus granulosus cyst. Anesthesist 1987; 36: 659-61.

22.- Ayles H M, Corbett E L, Taylor I, Cowie A G, Bligh J, Walmsley K, et al. A combined medical and surgical approach to hydatid disease: 12 years' experience at the Hospital for Tropical Diseases, London. Ann R Coll Surg Engl 2002;
84: $100-5$.

23.- Zlitni M, Ezzaouia K, Lebib H, Karray M, Kooli M, Mestiri M. Hydatid cyst of bone: diagnosis and treatment. World J Surg 2001; 25: 75-82.

24.- Tsagozis P, Brosjö O. Giant hydatid cyst of the pelvis, femur and retroperitoneal space: surgical treatment with extended hemipelvectomy. BMJ Case Rep 2015. doi: 10.1136/bcr-2015-209715.

25.- Torgerson P R, Deplazes P. Echinococcosis: diagnosis and diagnostic interpretation in population studies. Trends Parasitol 2009; 25 : 164-70. doi: 10.1016/j.pt.2008.12.008.

26.- Neumayr A, Troia G, de Bernardis C, Tamarozzi F, Goblirsch S, Piccoli L, et al. Justified concern or exaggerated fear: the risk of anaphylaxis in percutaneous treatment of cystic echinococcosis - a systematic literature review. PLoS Negl Trop Dis 2011; 5: e1154. doi: 10.1371/journal.pntd.0001154.

27.- Steinmetz S, Racloz G, Stern R, Domínguez D, Al-Mayahi M, Schibler M, et al.

Treatment challenges associated with bone echinococcosis. J Antimicrob Chemother 2014; 69: 821-6. doi: 10.1093/jac/dkt429.

28.- Loos J A, Dávila V A, Rodrígues C R, Petrigh R, Zoppi J A, Crocenzi F A, et al. Metformin exhibits preventive and therapeutic efficacy against experimental cystic echinococcosis. PLoS Negl Trop Dis 2017; 11 (2): e0005370. doi: 10.1371/journal.pntd.0005370.

29.- Bonifacino R, Dogliani E, Craig P S. Albendazole treatment and serological followup in hydatid disease of bone. Int Orthop 1997; 21: 127-32.

30.- Turtas S, Viale E S, Pau A. Long-term results of surgery for hydatid disease of the spine. Surg Neurol 1980; 13: 468-70. 